\title{
Conocimiento Especializado del Profesor de Matemáticas acerca del Infinito
}

\section{Mathematic Teachers' Specialized Knowledge about Infinity}

\author{
Miguel Montes* \\ José Carrillo**
}

\begin{abstract}
Resumen
El infinito es un concepto subyacente a multitud de conceptos presentes en la matemática escolar. En este artículo exploramos el conocimiento del profesor acerca del infinito desde la óptica del conocimiento profesional que usa para tratarlo en el aula. Para ello se usa la discusión de viñetas de simulación de situaciones en las que se reflejan diferentes tipos de razonamiento usando el concepto. Utilizaremos el modelo MTSK para tener una visión detallada de dicho conocimiento puesto en uso, mostrando que conocer el infinito en un sentido especializado implica conocerlo desde una perspectiva matemática y didáctica.
\end{abstract}

Palabras clave: MTSK. Infinito. Conocimiento profesional. MKT. Viñetas.

\begin{abstract}
Infinity is a concept underlying many concepts present in school mathematics. In this article, we explore teachers' knowledge about infinity from the perspective of the professional knowledge, which is used to deal with infinity in a classroom. For that purpose, we use the discussion of vignettes of simulated situations where different kinds of understandings of infinity are present. We will use the MTSK model to have a detailed insight of that knowledge, showing that knowing infinity, in a specialized sense, means knowing it both, from a mathematical and didactical perspective.
\end{abstract}

Keywords: MTSK. Infinity. Professional Knowledge. MKT. Vignettes.

\section{Introducción}

El infinito se ha estudiado en Educación Matemática desde diversas ópticas, desde los trabajos de Fischbein, Tirosh y Hess (1979), hasta los últimos avances en la exploración del aprendizaje usando software (KIDRON; TALL, 2015). Sin embargo, el foco de estas investigaciones suele ser el desarrollo del aprendizaje (BELMONTE, 2009), mientras que en

\footnotetext{
*Doctor en Didáctica de las Matemáticas por la Universidad de Huelva (UHU). Profesor sustituto Interino de Didáctica de la Matemática, Universidad de Huelva (UHU), Huelva, España. Dirección postal: Facultad de Ciencias de La Educación, Campus El Carmen, Avenida del 3 de Marzo, s/n, 21071. Huelva, España. E-mail: miguel.montes@ddcc.uhu.es.

${ }^{* *}$ Doctor en Filosofía y Ciencias de la Educación por la Universidad de Sevilla (US). Catedrático de Universidad de Didáctica de la Matemática, Universidad de Huelva (UHU), Huelva, España. Dirección postal: Facultad de Ciencias de La Educación, Campus El Carmen, Avenida del 3 de Marzo, s/n, 21071. Huelva, España. E-mail: carrillo@uhu.es.
} 
escasas investigaciones el interés se centra en el conocimiento del infinito que el profesor usa en el aula.

Asumimos que el profesornecesita conocer las matemáticas en un sentido diferente alde otro profesional usuario de las mismas, como pudiera ser un ingeniero. Esta diferenciaconcierne, también, a los diversostipos de reflexión que el profesor debe establecer sobre la disciplina que enseña. Con vista al concepto matemático aquí abordado, el infinito, nos preguntamos ¿qué tipo de reflexiones puede establecer un profesor sobre el infinito?

Coincidimos con Hannula, Pehkonen, Maijala y Soro (2006, p.1) en que "la mayoría de los niños de primaria están muy interesados por el infinito, y disfrutan reflexionando sobre el concepto, si el profesor está listo para ello”, reflexión que extendemos a los estudiantes de secundaria. Sin embargo, pese a que creemos que el conocimiento que el profesor pudiera activar para discutir el infinito per se puede dar lugar a un campo de estudio interesante, aquí nos centramos en el conocimiento acerca del infinito que resulta útil al profesor para enseñar contenidos matemáticos estandarizados y presentes en los diferentes currículos.

El profesor puede encontrar el infinito como elemento involucrado en diferentes procesos de enseñanza y aprendizaje, en diferentes contextos matemáticos (MAMOLO; ZAZKIS, 2008), y en diversos niveles educativos, y por tanto debería conocerlo y tenerlo en cuenta. En este artículo, mostramos diferentes formas en las que un profesor puede reflexionar, conocer y usar el infinito, categorizándolas a través del modelo de conocimiento especializado del profesor de matemáticas -MTSK-(CARRILLO et al.,2013). Para esto, en los antecedentes teóricos se profundiza en el modelo de conocimiento profesional usado, y se realiza una revisión de antecedentes de investigación relativa al infinito como objeto de conocimiento humano. Posteriormente, mostramos cómo la herramienta metodológica usada para extraer información, las viñetas, permiten la emergencia de conocimiento del profesor sobre el infinito, para finalizar discutiendo los diferentes aspectos y formas en que se puede conocer el infinito como profesor.

\section{Fundamentos teóricos}

En esta sección abordamos los dos pilares teóricos de esta investigación.Por un lado, el conocimiento profesional, donde introducimos el modelo de Conocimiento Especializado del Profesor de Matemáticas -MTSK- (CARRILLO, et al., 2013). Por otro lado,las reflexiones sobre la naturaleza de la cognición del infinito, y el tipo de consideración dada a los profesores en las investigaciones que pretenden profundizar en ésta. 


\subsection{Conocimiento Profesional: MTSK}

El conocimiento del profesor de matemáticas ha sido foco de estudio desde los años 1980, con las aportaciones de Shulman (1986,1987), continuadas por multitud de investigadores, entre los que destaca la propuesta de Ball, Thames y Phelps (2008), el Conocimiento Matemático para la Enseñanza -MKT-. Esta propuesta se ha usado en multitud de investigaciones que abordan el conocimiento del profesor desde diferentes ópticas, a la vez que ha sido criticada por algunas dificultades derivadas de la categorización en subdominios propuesta (e.g. SILVERMAN; THOMPSON, 2008; FLORES; ESCUDERO; CARRILLO, 2013). Desde nuestra perspectiva, una de las aportaciones que hace el modelo MKT es el reconocimiento de lo especializado del conocimiento del profesor de matemáticas, especialización que se entiende como la exclusividad de algunos de los conocimientos que este profesor posee. En la investigación que aquí plasmamos, usamos una noción de especialización diferente, integrada en el modelo de conocimiento profesional que usamos (Figura 1), el MTSK (CARRILLO et al., 2013).

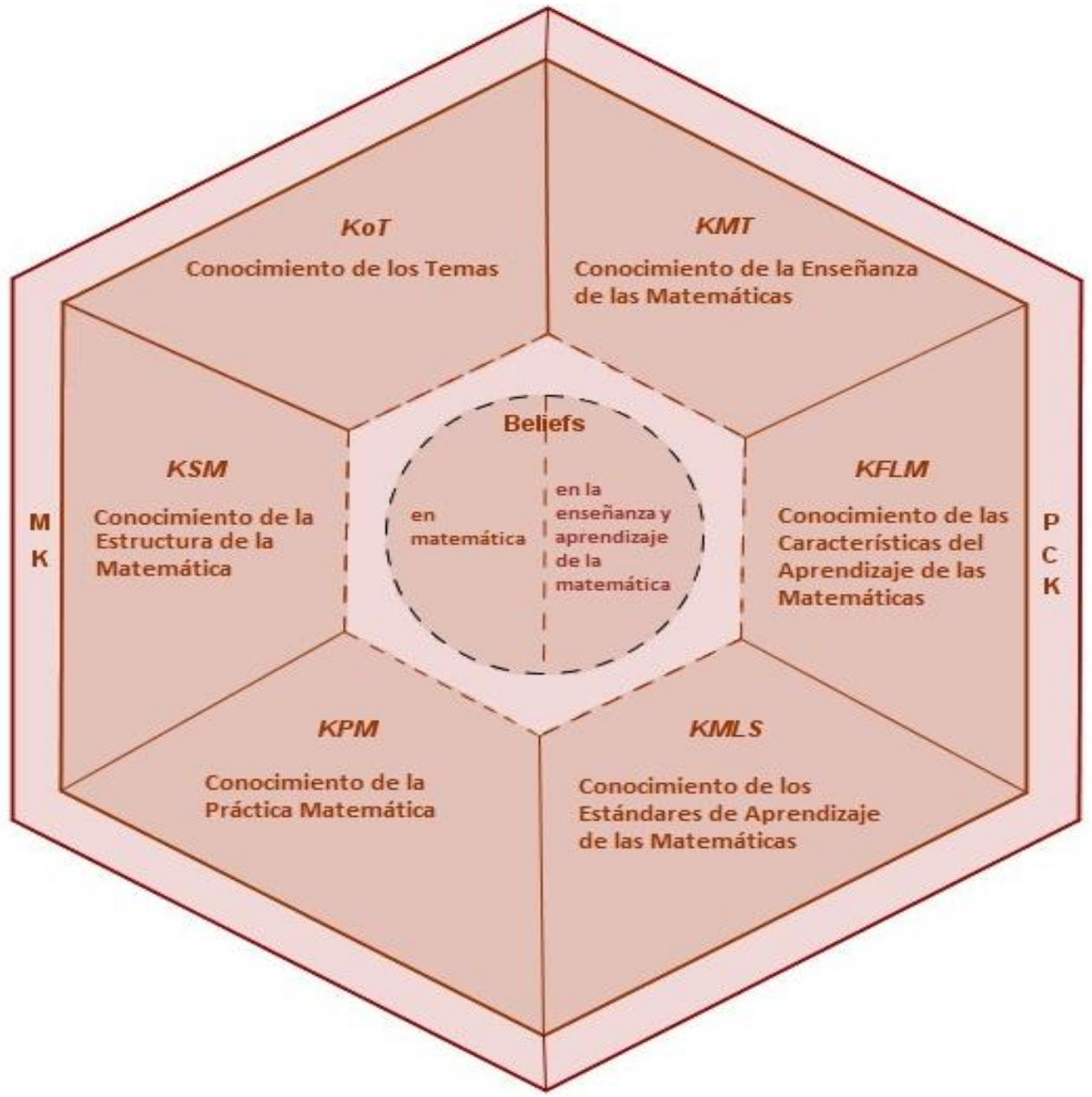

Figura 1 - Conocimiento Especializado del Profesor de Matemáticas, MTSK Fuente: Carrillo et al., 2013 
Desde esta perspectiva de la especialización, el conocimiento de un profesor es especializado en términos del tipo de conocimiento que un profesor de matemáticas necesita para enseñar, que tiene especificidades derivadas de la propia matemática. Así, mientras en el modelo MKT se consideraba como especializado aquel conocimiento exclusivo del profesor, en el MTSK, consideramos especializado el conocimiento ligado a las matemáticas que requiere un profesional de la enseñanza de las mismas. Esta ligazón con las matemáticas excluye considerar conocimiento pedagógico general como especializado del profesor de matemáticas, ya que es especializado de cualquier docente.El modelo MTSK propone considerar dos dominios del conocimiento profesional, de forma similar a Shulman (1986), separando conocimiento didáctico del contenido y conocimiento de la materia, en este caso, conocimiento matemático. Asimismo, en este modelo se considera las creencias como un elemento que influye sobre el conocimiento que los profesores desarrollan acerca del contenido. A continuación, describimos los subdominios de MTSK, primero los relativos al conocimiento matemático y, posteriormente, los englobados en el conocimiento didáctico del contenido, que permitirán dar sentido a las posteriores reflexiones sobre el conocimiento del profesor acerca del infinito, en base a las diferentes naturalezas de este conocimiento.

\subsubsection{Subdominios del conocimiento matemático}

El primer subdominio que propone el modelo es el conocimiento de los temas (KoT). Entendemos como tema las componentes de cada una de las áreas propuestas por el NCTM (2000) (números y operaciones, álgebra, geometría, medida, análisis de datos, y probabilidad). Estos temas no incluyen al infinito, pero se puede encontrar su presencia de forma transversal a algunos de ellos. Dentro de este conocimiento de los temas, podemos encontrar el conocimiento de diferentes operaciones, propiedades y sus fundamentos, de definiciones de los conceptos, de la fenomenología (FREUDENTHAL, 1983) del tema, o de diversos significados asociados a los conceptos del tema (e.g. en el caso de las fracciones, LLINARES; SÁNCHEZ, 1990). En resumen, significa conocer el tema, o conceptos dentro del mismo, con una profundidad mayor que otrosusuarios de las matemáticas (no docentes) pudieran requerir, siendo, por tanto, conocimiento de carácter especializado, que incluye el conocimiento que dicho usuario potencialmente pudiera adquirir.

En segundo lugar, se propone el conocimiento de la estructura matemática (KSM), que engloba un entendimiento del tópico matemático desde la perspectiva de un contexto matemático más amplio (KILPATRICK, 2008), permitiendo al profesor relacionar temas 
diferentes, o establecer conexiones entre conceptos más alláde los pretendidos en una sesión concreta. Este subdominio no incluye, de manera concreta, las especificidades del conocimiento sobre un tema específico, si bien dichas especificidades pueden influir profundamente en cómo un tema está relacionado con otro. En este subdominio se considera, por tanto, la consciencia de las conexiones, en el sentido de Fernández et al. (2010), de carácter interconceptual, así como las que responden a procesos de complejización o simplificación del contenido (MONTES; RIBEIRO; CARRILLO, en prensa).

Finalmente, se considera el conocimiento de la práctica matemática (KPM), que enlaza con la propuesta de Schwab (1978), de conocimiento sintáctico del contenido. Aquí, están incluidos los saberes del profesor relativos a los procedimientos de establecimiento de la validez en matemática, tipos de demostraciones, diferentes tipos de razonamientos heurísticos, así como la familiaridad con los procesos de modelización matemática y generalización.

Los tres subdominios anteriores, entendidos en su conjunto (con una visión holística del conocimiento matemático del profesor), dan una visión completa del conocimiento matemático del profesor, abarcando aquellos temas concretos abordados en el aula, desde una perspectiva de profundización en el tema (KoT), la interrelación de unos temas con otros, dando sentido a un cuerpo matemático sin partes aisladas (KSM), y las reglas de articulación de la actividad matemática (KPM)

\subsubsection{Subdominios del conocimiento didáctico del contenido}

MTSK contempla tres subdominios propios del conocimiento didáctico del contenido, siguiendo la subdivisión propuesta por Ball, Thames y Phelps (2008), pero cambiando el foco de los subdominios propuestos por estos autores para dar una mayor concreción, así como para hacer más relevante el papel de las matemáticas en estos.

En primer lugar, el conocimiento de la enseñanza de las matemáticas (KMT) es aquel conocimiento que el profesor posee y usa para hacer que sus alumnos comprendan mejor el contenido. Esto incluye conocimiento de recursos de enseñanza de las matemáticas (e.g. Geogebra, ábaco, regletas); de teorías (formales o informales) de enseñanza de las matemáticas; o de estrategias de enseñanza del contenido con las que conseguir fomentar el tipo de aprendizaje deseado.

En segundo lugar, se propone el subdominio del conocimiento de las características de aprendizaje de las matemáticas (KFLM). Este subdominio engloba las dimensiones cognitiva y epistemológica del conocimiento matemático del profesor (SCHEINER, 2015) acerca de sus 
alumnos, así como el conocimiento de aspectos emocionales inherentes al aprendizaje matemático que puedan condicionar cómo interactúan los estudiantes con el contenido matemático (CHARALAMPOUS; ROWLAND, 2013). En este subdominio podemos encontrar el conocimiento que posibilita desarrollar las habilidades que permiten al profesor mirar con sentido el aprendizaje de sus alumnos (FERNÁNDEZ; LLINARES; VALLS, 2012), estando éstas profundamente relacionadas con el contenido matemático involucrado.

Por último, se considera que un profesor puede tener conocimiento de los estándares de aprendizaje matemático (KMLS). Este subdominio amplía las consideraciones puramente curriculares de Shulman (1986) y Ball, Thames y Phelps (2008), para considerar la posibilidad de que el profesor pudiera conocer referentes estandarizados de lo que se debe aprender en determinado curso y cómo. Así, este subdominio añade, al conocimiento curricular, el conocimiento de estándares de asociaciones de profesores (e.g. NCTM, 2000), las planificaciones docentes internas de los centros, o el conocimiento de cómo profesores expertos que se pueden tomar como referente organizan sus cursos.

\subsubsection{Creencias y Concepciones}

El modelo que usamos como fundamento teórico de esta investigación considera las creencias y concepciones como elemento que permea, condiciona y complementael conocimiento del profesor, tanto en la forma y contenido de este, como en el uso que da el profesor a dicho conocimiento. Las creencias engloban "las verdades incontrovertibles sostenidas por cada individuo, derivadas de la experiencia o de la fantasía, con una fuerte componente afectiva y evaluativa". Por otro lado, las concepciones son entendidas como "las estructuras subyacentes a los conceptos, teniendo una naturaleza esencialmente cognitiva" (PONTE, 1994, p.199). En el caso del modelo de conocimiento del profesor que nos ocupa, las creencias y concepciones son relativas a la enseñanza y el aprendizaje de las matemáticas, así comoa la naturaleza de la propia matemática (ERNEST, 1991), o a conceptos concretos, como el límite (SIERPINSKA, 1987).

En este artículo, MTSK nos permitirá profundizar en el significado de la comprensión de un concepto como el infinito, que subyace a multitud de conceptos tanto de secundaria, bachillerato, y cursos universitarios de forma evidente, como de los primeros años de escolarización, si bien en estos es necesario realizar un análisis a nivel curricular y epistemológico más profundo para determinar la presencia del infinito en estos conceptos (e.g. LIÑÁN; MONTES; CONTRERAS, 2015). 


\subsection{Infinito}

Existe una amplia tradición de estudio y reflexión acerca del infinito por parte del ser humano, desde la discusión planteada por Aristóteles de las paradojas de Zenón, hasta el desarrollo de la teoría de transfinitos (CANTOR, 1932) o el análisis no estándar (ROBINSON, 1966). En particular, son interesantes los estudios centrados en cómo se ha desarrollado la cognición humana a lo largo de la historia (MORENO; WALDEGG, 1991; KLEINER, 2001), para dar sentido a cómo se desarrolla la cognición de los individuos, así como los trabajos que se centran en el desarrollo individual (e.g. FISCHBEIN; TIROSH; HESS,1979;PENALVA, 1996; LAKOFF; NUÑEZ, 2001), o en el desarrollo de estrategias de enseñanza y su impacto en el aprendizaje (e.g. TALL; SCHWARZENBERGER, 1978; KIDRON; TALL, 2015). Actualmente, la definición de infinito aceptada es la propuesta por Cantor (1932), como propiedad de aquellos conjuntos que pueden ponerse en correspondencia biunívoca con un subconjunto propio de sí mismos. En este sentido, puede estudiarse la finitud o no de un objeto matemático, estudiándolo como conjunto (e.g. una sucesión estudiada como el conjunto de elementos de la misma).

Una de las caracterizaciones habitualmente usadas en la investigación en educación matemática, sobre el infinito, es la diferenciación entre infinito potencial y actual (DUBINSKY et al., 2005). Esta caracterización está ligada a la forma de comprender el concepto, ya sea desde una perspectiva ligada a lo procesual del contexto en el que emerge, denominándose infinito potencial, o desde la consideración del proceso ya completado, el infinito actual.

En un proceso de revisión de literatura de investigación en educación matemática sobre el infinito (MONTES, 2015), hemos observado que existe una limitada cantidad de trabajos enfocados a la comprensión del infinito que presten atención al profesor como profesional de la enseñanza de las matemáticas; la mayoría lo consideran como un aprendiz del que se puede esperar un nivel avanzado de comprensión (e.g. DUBINSKY et al., 2005; YOPP; LINDAMAN; BURROUGHS, 2011). La línea de estudio que reflejamos aquí implica considerar que el profesor conoce el infinito no sólo como aprendiz, sino también como gestor del aprendizaje de sus alumnos. Así, los estudios de corte cognitivo serán útiles para determinar el tipo de comprensión que el profesor posee a nivel matemático, pero queremos profundizaren qué conoce el profesor acerca de la enseñanza y aprendizaje del infinito. De igual modo, las investigaciones que contemplan las creencias, concepciones y actitudes del profesor hacia este concepto también serán relevantes (e.g. SIERPINSKA, 1987), ya que éstas 
están imbricadas de forma natural con el conocimiento y con cómo y cuándo se pone éste en juego.

\section{Metodología}

El diseño de esta investigación esun estudio de casode carácter instrumental (BASSEY, 1999) a través del cualhemos profundizado en la naturaleza del conocimiento del infinito que el profesor activa en su enseñanza. El profesor estudiado (de pseudónimo Aarón) es Licenciado en matemáticas,con8 años de experiencia docente en secundaria, eimparte clase en España, en el primer curso de la secundaria (alumnado de 13 años), y en el curso previo al acceso a la Universidad (alumnado de 17 y 18 años), de manera que tuvimos la posibilidad de observarle impartir clase en dos nivelessignificativamente diferentes. En la entrevista previa, identificamos a Aarón como un profesor con el potencial de brindar información potente para este estudio, dado que no sólo mostró una actitud positiva hacia la investigacióne interés por el concepto de infinito, sino también una base de conocimiento matemático que le permitía generar una discusión interesante de situaciones donde el infinito está involucrado.

El proceso de recogida de información contempló tres instrumentos: un cuestionario amplio, que permitió una primera toma de contacto del profesor con la temática a abordar (a la vez que permitió a los investigadores tener una primera toma de contacto con su conocimiento); la audiograbación de 13 sesiones, con diferentes temáticas: representación de funciones, derivación, integración, y números decimales y periódicos; y cuatro entrevistas semiabiertas y semiestructuradas con tres temáticas: la discusión de situaciones matemáticas en las que el infinito está involucrado; la discusión de siete viñetas (JAKOBSEN et al.,2012) diseñadas con base en trabajos sobre aprendizaje del infinito, sobre los que se realizaban cuestionamientos al profesor ligados a la matemática, su enseñanza y su aprendizaje; y la profundización en algunos eventos sucedidos en las clases a las que se asistió. Con estos instrumentos se alcanzó una saturación de información que permitió mostrar evidencias de los resultadosobtenidos, a través de un análisis de contenido (BRYMAN, 2001)

En este artículonos centramos en la discusión de las siete situaciones o viñetas que permitieron profundizar en cada uno de los subdominios de conocimiento especializado de Aarón, a través de la discusión de situaciones en las que hipotéticos alumnos mostraban diferentes formas de aproximarse a la comprensión del infinito. Estas viñetas fueron diseñadas de manera que contemplaran diferentes patrones cognitivos observables en alumnos de 
secundaria (BELMONTE; SIERRA, 2010). En el Cuadro 1 se presentan las viñetas discutidas:

\begin{abstract}
Situación 1
Un profesor está explicando en una clase elementos relacionados con los números, yestablece la correspondencia biunívoca entre los naturales y los pares.Un alumno dice: ¿Cómo va a haber los mismos números pares que los naturales? ¡Pero si los pares están dentro de los naturales!
\end{abstract}

\title{
Situación 2
}

En otra clase, el profesor trabaja la no numerabilidad de los reales, y un alumno pregunta:Aunque los reales no sean numerables, los naturales y los reales siguen teniendo el mismo número de elementos, ¿no?

El profesor responde:¿Por qué lo preguntas?

A lo que el alumno afirma: Como ambos tienen infinitos elementos...

\section{Situación 3 \\ Profesor: Tres líneas rectas no siempre se cortan en un mismo punto, ¿no? \\ Alumno: Depende del tamaño del punto, ¿no?}

\section{Situación 4}

Un profesor propone el siguiente ejercicio en clase:

Ordenad por tamaño los siguientes conjuntos:

a) Número de estrellas

b) Número de granos de arena en la Tierra

c) Números naturales $\{1,2,3,4,5 \ldots\}$

d) Número de puntos que caben en un cuadrado de $10 \mathrm{~cm}$ de lado

e) Número de células que forman el cuerpo humano

(BELMONTE, SIERRA (2011))

Uno de los alumnos responde: Este ejercicio es imposible, en ningún caso soy capaz de contar cuántos hay, así que todos son infinitos, y no puedo compararlos.

\section{Situación 5}

Un profesor propone el siguiente ejercicio en clase:

Imaginad un número. Divididlo entre dos. El resultado, divididlo de nuevo entre dos, y así sucesivamente. ¿Qué resultado dará al final?

Un alumno responde:No se sabe, porque no sabemos cuándo parar.

\section{Situación 6}

Un profesor, en la clase de introducción a las series, plantea la siguiente cuestión:

$\mathrm{Si}$ estoy en un punto, imaginaos que el origen, y doy un paso de medio metro, luego uno de un cuarto de metro, otro de un octavo, y así sucesivamente, ¿dónde acabaré?

Un alumno responde: Pues a ver, profesor, si vas dando muchos pasos, muchos pasos, por muy pequeños que sean, te pasarás del 1 metro, te pasarás de los 2 metros, y así con cualquier medida, así que te irás al infinito.

\section{Situación 7}

Un alumno, después de una clase sobre teoría de conjuntos, pregunta:

Profesor, yo tengo una duda, a ver, como $[0,1)$ está acotado y $[0, \infty)$ no lo está, entonces en el segundo conjunto hay más elementos que en el primero, ¿no?

\section{Cuadro 1 - Viñetas de discusión}

Fuente: elaboración propia

Estas situaciones, propuestas bajo la hipótesis de que emergían en una clase de $4^{\circ}$ de ESO, están basadas en diferentes modelos intuitivos de comprensión del infinito (BELMONTE; SIERRA, 2011). Para el diseño, se hizo un análisis en profundidad del currículo español de manera que los contenidos se adecuaran a la realidad escolar (MONTES, 2015). La situación 1 está basada en el modelo intuitivo de inclusión (FISCHBEIN; TIROSH; 
HESS, 1979; FISCHBEIN, 1987; TIROSH; FISCHBEIN; DOR, 1985), ligado al principio holístico el total es mayor que sus partes. En la propia situación se indica que el profesor ha mostrado la igualdad cardinal entre ambos conjuntos, lo que causa una contradicción para el alumno, debido a la lógica conjuntista que subyace al razonamiento, válida en conjuntos finitos.

La situación 2 se desarrolló atendiendo al modelo de aplanamiento (D’AMORE; ARRIGO, 2006), en el que el sujeto obvia las características cardinales de los conjuntos, concibiendo todos los entes infinitos como iguales, atribuyéndoles cierto carácter indefinido que genera la equivalencia entre todos ellos.

La situación 3 refleja un resultado informalmente conocido como 'teorema del punto gordo', que se basa en la atribución de dimensiones o una naturaleza material a un punto geométrico (BELMONTE; SIERRA, 2011), generando un modelo en el que la recta, por ejemplo, se entiende como un modelo de collar de puntos (D'AMORE; ARRIGO, 2006). Este tipo de razonamiento genera problemas de comprensión, ya que conceptualiza el punto como un círculo, lo que lleva a problemas de comparación entre elementos geométricos.

Las situaciones 4 y 5 se basanen el modelo de indefinición (BELMONTE; SIERRA, 2011), en el que se sitúan muchos alumnos de esta edad, relacionando el infinito con la carencia de la propiedad tener final, lo cual se asocia a cierta indefinición en las propiedades de los elementos matemáticos involucrados, siendo la respuesta habitual en los alumnos que se enfrentan a la situación: no se sabe.En estos dos casos, mostramos varios contextos diferentes, el primero, formados por elementos sujetos a un conteo, que por su propia naturaleza no pueden ser infinitos (granos de arena en la Tierra, células en el cuerpo humano), o que han de serlo (números naturales, puntos en un cuadrado), o que están sujetos a discusión (cantidad de estrellas), y el segundo, un proceso infinito.

La situación 6 está ligada a la sistemática designación de infinito como el resultado de la suma de cualquier cantidad infinita de objetos matemáticos. Este patrón de pensamiento, explorado por Belmonte (2009), está ligado a la propiedad arquimediana, que afirma que la suma de infinitas cantidades finitas da un resultado infinito, y que requiere la consideración de elementos no arquimedianos para su contra-ejemplificación.

La última situación refleja un modelo de pensamiento derivado de asociar la propiedad de finitud a lo acotado y la de infinitud a lo no acotado (BELMONTE; SIERRA, 2011).De igual manera, dada la naturaleza del contenido, existía la posibilidad de centrar la discusión en qué hacer si se pretende comparar el intervalo $[0,1)$ con los reales positivos, en pos de 
explorar si el profesor considera el infinito como un elemento sujeto a corporeización (LAKOFF; NUÑEZ, 2001).

Se decidió no acompañar de un registro gráfico a cada una de las viñetas para no condicionar el tipo de reflexión que pudiera emerger de la discusión de las mismas, en particular, para evitar inducir un razonamiento de tipo potencial por dicho registro (LAKOFF; NUÑEZ, 2001).

Al profesor se le plantearon varias preguntas (Cuadro 2), que permitían explorar diferentes facetas de su conocimiento especializado como profesor de matemáticas.

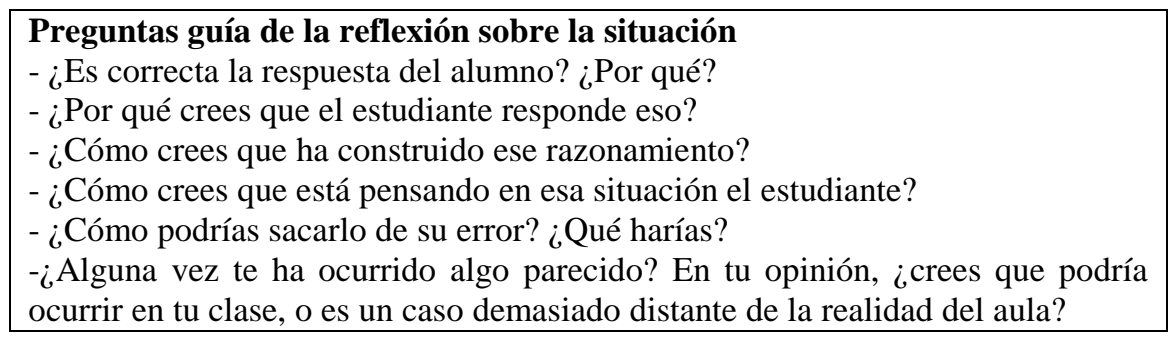

Cuadro 2 - Preguntas para la discusión de viñetas

Fuente: elaboración propia

La primera pregunta está orientada a la exploración de los subdominios del conocimiento matemático. Dependiendo de la respuesta dada por el profesor, se podría explorar un subdominio u otro, en pos de comprender la profundidad del conocimiento de los elementos discutidos (KoT), reflejando esta profundidad una forma de tratar el infinito; asimismo, se podía explorar la conexión del contenido con otros y observar si en diferentes contenidos relacionados (KSM) se daba el mismo tratamiento al infinito; finalmente, existía la posibilidad de tratar temas relacionados a la forma de hacer y expresar las matemáticas (KPM), con elementos como todo, algún, o elementos relacionados con la generalización de afirmaciones.

Posteriormente, se pasaba a varias preguntas relacionadas con el estudiante, que nos permitirían explorar el conocimiento del profesor de las características de aprendizaje de sus alumnos (KFLM), buscando posibles justificaciones desde diferentes perspectivas (práctica, matemática, cognitivo-intuitiva) de la respuesta del alumno. Tras esto, se induce al profesor a desplegar su conocimiento de la enseñanza de las matemáticas, en particular de esta situación, para profundizar en cómo abordaría la situación desde las acciones que llevaría a cabo (KMT), lo cual podría generar reflexiones ligadas a materiales manipulativos, estrategias de enseñanza, o dinámicas para conseguir un aprendizaje significativo de la situación. Finalmente, se abre la posibilidad de que el profesor comente experiencias propias, así como de que critique la plausibilidad de esta situación, sirviendo esta crítica como herramienta de 
triangulación adicional de la viñeta, en términos de su realismo. No se plantearon preguntas ligadas al conocimiento de los estándares de aprendizaje en matemáticas (KMLS), ya que en una de las entrevistas se había profundizado en este tema.

\section{Análisis de la discusión de las viñetas}

La discusión con el profesor, de las viñetas mostradas, generóuna sesión completa de entrevista, algunos de cuyos extractos mostramos aquí, de manera que podamos abundar en las diversas formas en las que el profesor puede conocer y usar el infinito al reflexionar sobre potenciales situaciones de clase. Por ejemplo, en torno a la situación 2, emergió la siguiente discusión entre Aarón (A), y el entrevistador (E):

A: Parece que la primera tendencia sería pensar: El alumno está razonando en $R$ de la misma forma que al comparar los naturales y los pares. [...]Parece que no tiene claro el concepto de infinito. Los niños en general tienden a pensar gráficamente y por ejemplo, al representar los conjuntos [como figuras cerradas y acotadas], esto ya da finitud a los conjuntos. Entonces, si $N$ es finito, $R$ es finito, porque se representan igual. Quizá sea un error incluso nuestro [de los profesores], de no pensar por qué lo hacemos así y de llevarnos siempre todo lo que hacemos a lo gráfico. [...]

E: ¿Y cómo harías para sacarlo de su error?

A: Me lo llevaría a la comparación de conjuntos infinitos, el $(0,1)$ y los reales, por ejemplo, demostrando que tienen los mismos de manera gráfica, pintándole la recta real "grande", y el intervalo encima. Desde ahí creo que podría empezar a trabajar.

(Extracto de la entrevista, Montes 2015)

Vemos, en este caso, cómo Aarón parece poseer conocimiento del infinito desde una perspectiva tanto matemática como didáctica del concepto. Vemos cómo asocia directamente el razonamiento del alumno a la comparación que se establece entre los números naturales y los números pares, quedando mediada la comprensión del alumno de la relación entre dos conjuntos infinitos por la relación entre otros dos conjuntos infinitos. Para realizar este razonamiento, Aarón usa su propio conocimiento del tema de conjuntos numéricos (KoT). Asimismo, genera una justificación posible de cómo el alumno llega a esta conclusión, en términos del tipo de dinámica de enseñanza que se sigue habitualmente. Atribuye a representaciones, habitualmente usadas, para mostrar las relaciones entre conjuntos numéricos la potencial generación de un obstáculo de comprensión de dichas relaciones. Este tipo de conocimiento mezcla tanto conocimiento de las características del aprendizaje (KFLM), ligados a la interacción de los alumnos con este tipo de representación de los conjuntos, como conocimiento de la enseñanza de las matemáticas (KMT), en particular sobre el uso de representaciones gráficas como vía para aproximarse a este tipo de conceptos. 
Finalmente, Aarón es capaz de esbozar una aproximación de cómo ayudar al alumno a superar su obstáculo, a través de la comparación de dos conjuntos del mismo cardinal, en cuya representación se requiere abstraer la infinitud de los reales, por ejemplo, representando la no acotación con flechas o puntos suspensivos.

A: Los granos de arena en la Tierra, como están acotados [la unión de todos ellos], y tienen cierto tamaño, son finitos, luego los puedes contar. Hablaría de una comparación, granos de arena con células, y la Tierra con el cuerpo humano.

E.: Me dices entonces que los granos de arena los puedes contar, pero ¿cómo? El alumno dice que al no poder contarlo es infinito.

A.: Es verdad. De hecho, su razonamiento me parece bien. Por lógica, si no puedes contarlos, son infinitos. Me parece de pura lógica, y por tanto no le diría que no. No puedes contarlos, luego son infinitos. [...] Y no lo sacaría de su error, de hecho.

E.: Sin embargo, en otros ejemplos en los que no puedes contar, me has dicho que son finitos.

A.: Pues me corrijo. Acepto el argumento. Es que si tienen claro que si no lo pueden contar, para ellos es infinito, en una situación tan física, tan cercana a la realidad, pues le diría que lleva razón. Quizá dependiendo del curso me plantearía otra respuesta [es decir, corregir al alumno], aunque tendría que dedicar mucho tiempo a buscar cómo tratar el tema en función del curso.

(Extracto de la entrevista, Montes 2015)

Este extracto resulta interesante por cómo el conocimiento matemático de Aarón se moviliza y parece adecuarse al contexto escolar en que se plantea la cuestión. En primer lugar, Aarón muestra conocimiento puramente matemático al afirmar que el cardinal de los granos de arena es finito, ya quecada uno de ellos tiene una dimensión finita mayor que cero (y que una cierta cantidad), y su unión es acotada. Muestra en esto conocimiento del tema (KoT) series numéricas. Sin embargo, al incidirse en la posibilidad de contarlos, el profesor acepta el argumento del alumno de que si es imposible contar físicamente el número de elementos de un conjunto, se le puede atribuir a este conjunto la característica de la infinitud. Por esta declaración, y por afirmaciones anteriores, tenemos indicios (FLORES; ESCUDERO; AGUILAR, 2013) de que no sólo parece aceptar el argumento del alumno, sino que parece adaptar su propia concepción del infinito a ésta forma de comprenderlo, ligado a la necesidad de interacción física con las matemáticas. Más aún, cuando durante una de las entrevistas se le pidió una definición del infinito, Aarón afirmó: para mí el infinito es la invención del hombre para explicar lo inexplicable. Siguiendo a SIERPINSKA (1987), p. 382), a Aarón se le asocia una tendencia intuitiva empírica, "que implica comprender que la matemática, y en particular sus axiomas y teoremas, deben ser hechos indiscutibles, intuitivamente aceptados”. En este caso,acepta a la construcción lógica de los alumnos,ya que desde su perspectiva las matemáticas deben estar basadas en dicha aceptación intuitiva. Sin embargo, Aarón parece estar abierto a considerar que en otros cursos se pueda esperar una forma diferente de comprensión del concepto de infinito, al menos en su plasmación en conceptos matemáticos 
concretos. Esto supone un indicio de que el profesor conoce ciertos estándares de aprendizaje relativos (KMLS) a este concepto, o al menos ha desarrollado unos ciertos estándares informales sobre qué debe aprenderse en cada etapa educativa sobre el infinito.

El posicionamiento del profesor con respecto al infinito se evidencia de forma más clara en la discusión de la sexta situación:

A: Esto es como el ejemplo del salto de la rana que va saltando hacia el borde del estanque, preguntando si llega o no llega. Me ha pasado, de empezar a explicarles las sucesiones, y hablar del primer salto, el segundo que es la mitad del anterior, el tercero que es la mitad del segundo, y así sucesivamente, y muchos alumnos dicen que sí llega, pero no están convencidos. [...] Luego te lo llevas a un terreno matemático, empiezas a dividir por dos, una y otra vez. ¿Llega, o no llega?

E: ¿Y llega o no llega?

A: No, no lo hace. [...] Llega al borde, pero no lo hace. En términos físicos, no debería alcanzarlo.

(Extracto de la entrevista, Montes 2015)

En este caso, Aarón afirma que él mismo comienza discutiendo el ejemplo de las sucesivas divisiones en clase, y tiene la experiencia de que los alumnos dan una diversidad de respuestas a este problema. Entendemos que dicha experiencia constituye la base de su conocimiento de las características de aprendizaje acerca del infinito (KFLM). Más interesante aún, resulta la forma que tiene Aarón de comprender el infinito, que se ve reflejada en este ejemplo. Al igual que en el extracto anterior, Aarón muestra su necesidad de que las matemáticas tengan relación con el mundo físico, y por tanto entiende que la rana, en términos físicos, no debería alcanzar el borde del estanque, ya que posiblemente esté considerando la variable temporal en el proceso. Esto refuerza la idea de que Aarón sigue una tendencia intuitiva empírica acerca del infinito, y más aún, que esta tendencia intuitiva empírica le lleva a comprender el infinito de forma potencial, fijándose en el proceso de sucesivo acercamiento al borde del estanque. Esta comprensión potencial del infinito, en este caso, es la suya propia, no la que atribuye a sus alumnos. Posteriormente se le preguntó cómo abordaría esa situación desde una perspectiva más manipulativa, a lo que respondió:

A: Tomaría un trozo de papel de un metro de longitud como referencia, y luego otra de medio metro de longitud, que pondría encima. Después de esto, otro de un cuarto de metro, que pondría a la derecha de la anterior, luego uno de un octavo. En algún momento les diría que, si se hace zoom sobre el proceso, volvemos a lo que esencialmente era el punto inicial, haciendo la comparación entre la pieza que queda y la mitad restante. Lo haría unas cuantas veces de esta forma para hacerles ver que nunca se podría pasar de este punto, llegues lo cerca que llegues.

(Extracto de la entrevista, Montes 2015)

Podemos observar cómo el profesor articula un abordaje metodológico del proceso aproximativo, desplegando una estrategia de enseñanza (KMT) basada en el cortado de papel 
(MONTES; CARRILLO, 2015). Es especialmente interesante observar cómo el planteamiento que el profesor hace de su posible explicación está muy condicionado por su comprensión del infinito. Observando la estrategia de enseñanza de forma detenida, hace hincapié en la particularización del proceso de adición de sucesivas mitades en los primeros términos, entendemos que para mostrar el proceso de funcionamiento, y, posteriormente, añade la idea de zoom. Esta idea es potente por dos motivos, en primer lugar añade la posibilidad de realizar el proceso de forma recurrente, lo cual permite comprender qué sucede en cada paso, y en segundo, es una forma de plasmar la infinitud del proceso, ya que el hecho de volver a la primera iteración en cierto paso implica poder volver en cualquier paso.

Sin embargo, esta aproximación a la suma de los elementos que hace el profesor se ve inequívocamente condicionada por su forma de comprensión del infinito. Antes mostramos evidencias de que Aarón comprende el infinito de forma potencial. En este caso, no sólo parece evidenciar dicho tipo de comprensión, al enfocar su reflexión en el aspecto procesual de la aproximación al borde, sino que diseña la actividad con el objetivo de hacer ver a los alumnos que nunca se podría pasar de este punto. Esta verbalización nunca implica la inclusión de la variable temporal, que conlleva, desde esta perspectiva potencial, la imposibilidad de alcanzar el extremo. En términos matemáticos, esta explicación equivale a considerar el conjunto de elementos de la sucesión de sumas parciales sin considerar el punto de acumulación de dicho conjunto. Este foco en la sucesión, sin tener en cuenta el punto de acumulación, es decir, esta visión potencial, puede generar en los alumnos una comprensión limitada a lo procesual de la aproximación (TALL; SCHWARZENBERGER, 1978). Por tanto, no sólo el conocimiento de los temas matemáticos (KoT) del profesor responde a estructuras potenciales de comprensión del infinito, sino que su conocimiento de la enseñanza de las matemáticas (KMT) relacionado con el infinito también está generado en base a este tipo de comprensión potencial.

Más aún, su comprensión del infinito como concepto, entendido como proceso, se filtra en aquellos conceptos en los que el infinito constituye el sustento epistemológico. En una entrevista posterior, sobre eventos sucedidos en su clase, encontramos lo siguiente:

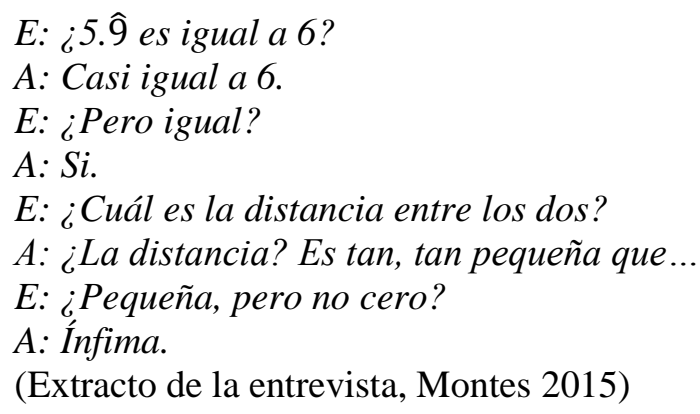


Vemos cómo Aarón lleva el mismo esquema de entendimiento potencial del concepto de infinito que vimos en el caso de la sucesión de las sumas parciales de potencias de 1/2 a este caso, el de la igualdad de 5. 9 y 6. La verbalización ínfima es equivalente, epistemológicamente, a infinitesimal (LAKOFF; NUÑEZ, 2001), y denota el hecho de dotar de un carácter variable a dicha distancia. Es decir, Aarón acepta que dicha distancia es menor que cualquier número conocido, pero a la vez que no es mayor que cero, convirtiéndola en un ente de naturaleza no arquimediana. Podemos inferir que el conocimiento o entendimiento del concepto de infinito en sí tiene un carácter transversal al conocimiento del profesor, relacionándose con diferentes conceptos matemáticos, mostrando indicios de conocimiento de la estructura matemática (KSM), en los que el profesor muestra una comprensión de tipo potencial.

\section{Conclusiones}

Múltiples autores han profundizado en el esfuerzo cognitivo que requiere alcanzar una comprensión del infinito, desde el punto de vista del aprendizaje. En este artículo, tomando esta asunción como punto de partida, hemos explorado el conocimiento del profesor del infinito, desde la óptica del conocimiento profesional, con una visión interpretativa, no basada en la evaluación del conocimiento del mismo, sino en la exploración de su complejidad.

Adquirir una comprensión profunda de este concepto implica una reflexión profunda que ha llevado siglos a la humanidad (MORENO; WALDEGG, 1991; KLEINER, 2001), dada la complejidad de dicha reflexión. Climent (2002), en la línea de Ponte (1994), establecía que el conocimiento del profesor posee una naturaleza compleja, dada la amplia variedad de tipos de conocimiento que éste pone en juego al enseñar. En nuestro caso, al estudiar el conocimiento del profesor acerca del infinito, desde la perspectiva de la especialización de dicho conocimiento derivada de su uso en la discusión de contextos ligados a la enseñanza y aprendizaje de las matemáticas, mostramos que existe una doble fuente de complejidad en el conocimiento del infinito que poseen los profesores.

La primera fuente está determinada por la complejidad que requiere comprender este concepto desde una perspectiva matemática, siendo ésta la línea habitual de las reflexiones establecidas desde la investigación en educación matemática al abordar el infinito. En el caso de Aarón, observamos una comprensión de tipo potencial del infinito, que le lleva a momentos de conflicto, en los que los argumentos de los alumnos le conducen a cuestionar afirmaciones, de índole matemática, que él mismo hace. 
La segunda fuente de complejidad del conocimiento del profesor acerca del infinito es la derivada del carácter especializado del conocimiento del profesor, es decir, del tipo de reflexión que, como docente, se establece en torno al concepto. Por ejemplo, reflexionar sobre la procedencia del razonamiento de los alumnos al tratar con el infinito, con base en el tipo de explicaciones recibidas, es un tipo de reflexión que podemos asumir que es exclusiva del docente, y dota de un grado de complejidad adicional al conocimiento de éste. En este mismo sentido, el modelo de Conocimiento Especializado del Profesor de Matemáticas, MTSK, se revela especialmente útil para caracterizar las diversas tipologías de conocimiento que el profesor usa.

Así, encontramos que el conocimiento del profesor acerca del infinito tiene carácter transversal en el conocimiento del profesor, adquiriendo la naturaleza de Gran Idea (KUNTZE, et al., 2010) presente en el conocimiento matemático del profesor en diferentes contextos matemáticos, lo que lo convierte en un elemento conector, ligándolo al conocimiento de la estructura matemática (KSM).

Hemos podido observar cómo el tipo de conocimiento que tiene el profesor acerca del infinito se refleja en su conocimientode aquellos conceptos que tienen a éste como sustento epistemológico (KoT). Más allá de esto, el tipo de comprensión que se tiene del infinito, como la potencial en el caso de Aarón, puede implicar una generación de ejemplos y estrategias de enseñanza (KMT) que induzcan en los alumnos dicho tipo de comprensión. Asimismo, la comprensión que posee un profesor acerca del aprendizaje de sus propios alumnos acerca del concepto estará potencialmente mediada por su propia comprensión del concepto, es decir, resulta difícil pensar que un profesor como Aarón, que entiende el infinito de una forma potencial, pudiera esperar en sus alumnos una comprensión de tipo actual de este concepto.

Queremos destacar el potencial que, a nuestro entender, tiene la discusión de viñetas en las que se observen situaciones de aula, reales, hipotéticas o pseudo-reales, cuyo diseño, en estos dos últimos casos, esté profundamente fundamentado en los distintos tipos de comprensión de los conceptos que han sido estudiados en la investigación en educación matemática. En el caso que nos ocupa, fueron especialmente útiles, ya que Aarón no había sido plenamente consciente de su interacción con el infinito durante su actividad docente, sólo lo había detectado en aquellos contextos donde éste era explícito (MONTES, 2015). Por tanto, la discusión de situaciones donde alumnos daban respuestas plausibles, pero inesperadas para él, ponían a Aarón en una situación de contingencia (ROWLAND et al., 2009) que le llevaba 
a movilizar su conocimiento en modos que, posiblemente, no hubiera movilizado anteriormente.

La línea de investigación aquí mostrada, acerca de la comprensión del infinito desde una perspectiva profesional, dota al área de educación matemática de una nueva línea que explorar y en la que profundizar. En este trabajo asumimos que conocer un concepto como el infinito, desde la óptica del conocimiento del profesor, no se limita a conocer el constructo matemático como tal, sino que un docente ha de tener una consciencia amplia de todo aquello que implica el infinito en contextos docentes: el concepto matemático, las relaciones que el infinito genera entre conceptos matemáticos, el tipo de aproximaciones docentes que se hace a estos conceptos, el tipo de aprendizaje esperable en alumnos, en particular el esperable en las situaciones docentes planteadas, o el nivel de comprensión esperable en determinada etapa educativa. En futuras investigaciones, pretendemos abordar el problema de la construcción, en profesores de Secundaria y Bachillerato, de un conocimiento del infinito que permita fomentar un aprendizaje de los alumnos que trascienda la visión potencial del mismo.

\section{Agradecimientos}

Los autores son miembros del proyecto de investigación Caracterización del Conocimiento Especializado del Profesor de Matemáticas (EDU2013-44047P), financiado por el Ministerio de Economía y Competitividad.

\section{Referencias}

BALL, D.L.; THAMES, M. H.; PHELPS, G. Content knowledge for teaching: what makes it special? Journal of Teacher Education, New York, v. 59, n.5, p. 389-407, nov./dic. 2008.

BASSEY, M. Case Study Research in Educational Settings. 1. ed. Scotland: Open University Press. 1999. $178 \mathrm{p}$.

BELMONTE MARTÍNEZ, J. L. Modelos Intuitivos y Esquema Conceptual del Infinito en Estudiantes de Educación Primaria, Secundaria Obligatoria, Bachillerato y Universidad.2009. 569 p. Tesis (Doctorado en Educación Matemática) - Departamento de Didáctica de la Matemática y Didáctica de las Ciencias Experimentales, Universidad de Salamanca, Salamanca, 2009.

BELMONTE MARTÍNEZ, J. L.; SIERRA, M. Modelos intuitivos del infinito y patrones de evolución nivelar. Revista Latinoamericana de Investigación en Matemática Educativa, México, v.14, n. 2.p. 139-171, jul. 2011.

BRYMAN, A. Social research methods. 1. ed. Nueva York: Oxford University Press, 2001.540 p. 
CANTOR, G. Gesammelte Abhandlungen Mathematischen und Philosophischen Inhalts. 1. ed. Berlin: Springer, 1932. 442 p.

CARRILLO, J. et al. Determining specialised knowledge for mathematics teaching. In: CONGRESS OF EUROPEAN RESEARCH IN MATHEMATICS EDUCATION, 8., 2013, Antalya. Proceedings of CERME 8.Antalya: ERME, 2013. p. 2985-2994.

CHARALAMPOUS, E.; ROWLAND, T. Mathematics Security and the individual.In: CONGRESS OF EUROPEAN RESEARCH IN MATHEMATICS EDUCATION, 8., 2013, Antalya. Proceedings of CERME 8. Antalya: ERME, 2013. p. 1299-1308.

CLIMENT, N. El Desarrollo Profesional Del Maestro De Primaria Respecto De Enseñanza De La Matemática. Un Estudio De Caso. 2002. 387 f. Tesis (Doctorado en Educación Matemática) Departamento de Didáctica de las Ciencias, Universidad de Huelva, Huelva, 2002.

D’AMORE, B. et al. El "sentido del infinito". Epsilon, España, v.22, n.65, p. 187-216, 2006.

DUBINSKY, E. et al. Some historical issues and paradoxes regarding the concept of infinity: an APOS analysis: part 2. Educational Studies in Mathematics, Utrecht, v. 60, n. 2, p.253-260, oct.2005.

ERNEST, P. The Phylosophy of Mathematics Education. 1. ed. Londres: Falmer Press, 1991.329 p.

FERNÁNDEZ, C.; LLINARES, S.; VALLS, J.Learning to notice students' mathematical thinking through on-line discussions. ZDM Mathematics Education, Berlín, v. 44, p. 747-759, oct.2012.

FERNÁNDEZ, S. et al. Re-defining HCK to approach transition. In: CONGRESS OF EUROPEAN RESEARCH IN MATHEMATICS EDUCATION, 7., 2010, Rzeszów. Proceedings of CERME 7.Rzeszów: ERME, 2010. p. 2640-2649.

FISCHBEIN, E.; TIROSH, D.; HESS, P. The intuition of infinity. Educational Studies in Mathematics, Utrecht, v. 10, n. 1, p. 491-512,feb.1979.

FISCHBEIN, E. Intuition In Science And Mathematics: An Educational Approach. 1. ed. Dordrecht: Reidel, 1987. 214 p.

FLORES-MEDRANO, E.; ÁVILA, D. I. E.; GONZÁLEZ, A. A. Oportunidades que brindan algunos escenarios para mostrar evidencias del MTSK. In: SIMPOSIO DE LA SOCIEDAD ESPAÑOLA DE INVESTIGACIÓN EN EDUCACIÓN MATEMÁTICA, 17., 2013, Bilbao. Investigación en Educación Matemática. Bilbao: Universidad de Bilbao, 2013. p. 275-282.

FLORES, E.; ESCUDERO, F. I.; CARRILO, J. A theoretical review of specialised content Knowledge. In: CONGRESS OF EUROPEAN RESEARCH IN MATHEMATICS EDUCATION, 8., 2013, Antalya. Proceedings of CERME 8. Antalya: ERME, 2013. p. 3055-3064.

FREUDENTHAL, H. Didactical Phenomenology Of Mathematical Structures. 1. ed. Nederlands: Springer, 1983. $578 \mathrm{p}$.

HANNULA, M. et al. Levels of students' understanding on infinity. Teaching Mathematics and Computer Science, Debrecen, v. 4, n. 2, p. 317-337, jan.2006.

JAKOBSEN, A. et al. Using practice to define and distinguish horizon content knowledge. In: INTERNATIONAL CONGRESS ON MATHEMATICAL EDUCATION, 12., 2012, Seoul. Proceedings of ICME 12.Seoul: ICME, 2012. p.4635-4644. 
KIDRON, I.; TALL, D. The roles of visualization and symbolism in the potential and actual infinity of the limit process. Educational Studies in Mathematics, Utrecht, v. 88, n. 2, p. 183-199, feb.2015.

KILPATRICK, J. A higher standpoint. In: INTERNATIONAL CONGRESS ON MATHEMATICAL EDUCATION, 11., 2008, Monterrey. Regular lectures of ICME 11. Monterrey: ICME, 2008. p. 2643.

KLEINER, I. History of the infinitely small and the infinitely large in calculus. Educational Studies in Mathematics, Utrecht, v. 45, n. 2-3, p. 137-174, mar. 2001.

KUNTZE, S. et al. Professional knowledge related to big ideas in mathematics: an empirical study with pre-service teachers. In: CONGRESS OF EUROPEAN RESEARCH IN MATHEMATICS EDUCATION, 7., 2010, Rzeszów. Proceedings of CERME 7.Rzeszów: ERME, 2010. p. 2717-2726.

LAKOFF, G.; NÚÑEZ, R. Where mathematics comes from: how the embodied mind brings mathematics into being. 1. ed. New York: Basic Books, 2001.512 p.

LIÑÁN, M.M., MONTES, M., CONTRERAS, L.C. Conocimiento sobre la recta de una maestra de tercer ciclo de educación primaria. In: SIMPOSIO DE LA SOCIEDAD ESPAÑOLA DE INVESTIGACIÓN EN EDUCACIÓN MATEMÁTICA, 19., 2015, Alicante. Investigación en Educación Matemática. Alicante: Universidad de Alicante, 2015. p. 335-342.

LLINARES, S.; SÁNCHEZ, V. Las creencias epistemológicas sobre la naturaleza de las matemáticas y su enseñanza y el proceso de llegar a ser un profesor. Enseñanza \& Teaching: revista interuniversitaria de didáctica, Salamanca, n. 8, p. 165-180, 1990.

MAMOLO, A.; ZAZKIS, R. Paradoxes as a window to infinity. Research in Mathematics Education, Londres, v. 10, n. 2, p. 167-182, aug.2008.

MONTES, M. Conocimiento Especializado del Profesor de Matemáticas Acerca del Infinito: un estudio de caso. 2015. 250 f. Tesis (Doctorado en Educación Matemática) -Departamento de Didáctica de las Ciencias y Filosofía, Universidad de Huelva, Huelva, 2015.

MONTES, M.; CARRILLO, J. What does it mean as a teacher to 'know infinity'? The case of convergence of series.In: CONGRESS OF EUROPEAN RESEARCH IN MATHEMATICS EDUCATION, 9., 2015, Praga. Proceedings of CERME 9.Praga: ERME, 2015. p. 3220-3226.

MONTES, M.; RIBEIRO, C.M.; CARRILLO, J. Towards a topology of mathematical connections in teacher knowledge. In: INTERNATIONAL CONGRESS ON MATHEMATICAL EDUCATION, 13., 2016, Hamburgo. Proceedings of ICME 13.Hamburgo: ICME, 2016.p. en prensa.

MORENO, L.; WALDEGG, G.The conceptual evolution of actual mathematical infinity. Educational Studies in Mathematics, Utrecht, v. 22, n.5, p. 211-231, oct. 1991.

NATIONAL COUNCIL OF TEACHERS OF MATHEMATICS. Principles and Standards for School Mathematics. 1. ed. Reston: NCTM, 2000. 402 p.

ROBINSON, A. Non-Standard Analysis. 1. ed. Amsterdam: Ed. North-Holland Publishing Co., $1966.308 \mathrm{p}$.

PENALVA, M.C. Estudio Sobre la Comprensión del Concepto de Número Cardinal de un Conjunto Infinito.1996. 120 f. Tesis (Doctorado en Educación Matemática) - Universidad de Valencia, Valencia, 1996. 
PONTE, J.P. Mathematics teachers' professional knowledge. In: INTERNATIONAL CONFERENCE FOR THE PSYCHOLOGY OF MATHE-MATICS EDUCATION, 18., 1994, Lisboa. Proceedings... Lisboa: Universidad de Lisboa, 1994.p. 195-210.

ROWLAND, T. et al. Developing Primary Mathematics Teaching: reflecting on practice with the knowledge quartet. 1. ed. Londres: SAGE, 2009.256 p.

SCHEINER, T. Lessons we have (not) learned from past and current conceptualizations of mathematics teachers' knowledge. In: CONGRESS OF EUROPEAN RESEARCH IN MATHEMATICS EDUCATION, 9., 2015, Praga. Proceedings of CERME 9. Praga: ERME, 2015. p. 3248-3253.

SCHWAB, J. J. Education and the structure of the disciplines. In: WESTBURY, I.; WILKOF, N.J. (Ed.).Science, Curriculum and Liberal Education. Chicago: Ed.University of Chicago Press, 1978. p. 229-272.

SHULMAN, L. Those who understand: knowledge growth in teaching. Educational Researcher, Washington, v. 15, n. 2, p. 4-14, feb.1986.

SHULMAN, L. Knowledge and Teaching: Foundations of the New Reform. Harvard Educational Review, Cambridge, v. 57, n.1, p. 1-22, 1987.

SIERPINSKA, A. Humanities students and epistemological obstacles related to limits. Educational Studies in Mathematics, Utrecht, v. 18, n. 4, p. 371-397, nov. 1987.

SILVERMAN, K; THOMPSON, P. W. Toward a framework for the development of mathematical knowledge for teaching. Journal of Mathematics Teacher Education, Dordrecht, v.11, n.6, p.499511, nov.2008.

TALL, D; SCHWARZENBERGER, R.L.E. Conflicts in the learning of real numbers and limits. Mathematics Teaching, Warwick, v. 82, p. 44-49, 1978.

TIROSH, D.; FISCHBEIN, E.; DOR, E. The teaching of infinity. In: CONFERENCE OF THE INTERNATIONAL GROUP FOR THE PSYCHOLOGY OF MATHEMATICS EDUCATION, 9., 1985. Utrecht. Proceedings of PME 9. Utrecht: PME, 1985, p. 501-506.

YOPP, D.; LINDAMAN, B; BURROUGHS, E.A. Why it is important for in-service elementary teachers to understand the equality $0.999 \ldots=1$. Journal of Mathematical Behavior, Amsterdam, $v$. 30, n.4, p. 304-318, dic. 2011.

Submetido em Abril de 2016. Aprovado em Novembro de 2016. 